Perspective

\title{
Diabetes and Obesity
}

Daphne Owens, Gerald H Tomkin *

Coillancarraig Lodge, Rocky Valley Drive, Kilmacanogue, Co Wicklow, Ireland; E-Mails: daowens@TCD.ie; TOMKING@tcd.ie

* Correspondence: Gerald H Tomkin; E-Mail: TOMKING@tcd.ie

Academic Editors: Roberto Cannataro, Diego A. Bonilla and Jorge Luis Petro Soto

Special Issue: Nutrition and exercise for Weight loss

OBM Integrative and Complementary Medicine Received: August 18, 2021

2021, volume 6 , issue 4

doi:10.21926/obm.icm. 2104048

Accepted: November 09, 2021

Published: November 22, 2021

\begin{abstract}
Obesity and Diabetes are leading causes of cardiovascular disease and mortality. They are closely linked because of insulin resistance. Hypertension and dyslipidaemia together with poor exercise drive atherosclerosis. Early treatment of blood sugar, dyslipidaemia and hypertension reverse the atherosclerosis process. Bariatric surgery and low calorie diets have in recent years been shown to reverse or improve blood sugars lipids and blood pressure. The risk of premature myocardial infarction and death should no longer be the desired outcome.
\end{abstract}

\section{Keywords}

Diabetes; atherosclerosis; dyslipidaemia: and low calorie diet's

\section{Summary}

Obesity and diabetes are leading causes of cardiovascular disease and mortality. They are closely linked because of insulin resistance. Hypertension and dyslipidaemia together with poor exercise, drive atherosclerosis. Early treatment of blood sugar, dyslipidaemia and hypertension reverse the

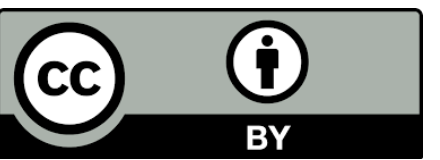

(C) 2021 by the author. This is an open access article distributed under the conditions of the Creative Commons by Attribution License, which permits unrestricted use, distribution, and reproduction in any medium or format, provided the original work is correctly cited. 
atherosclerosis process. Bariatric surgery and low calorie diets have, in recent years, been shown to reverse or improve blood sugars, lipids and blood pressure. The risk of premature myocardial infarction and death should no longer be the acceptable outcome.

The trajectory for incidence of diabetes has been upwards in the last 50 years. The trajectory for obesity has followed the diabetes pattern or is it the other way round and the rising incidence of obesity has spawned the rise in diabetes. Diabetes is a very damaging disease and is associated with early mortality. A surprise therefore that the trajectory for increase in age of the population mirrors both obesity and diabetes [1]. Ninety percent of patients who have diabetes have insulin independent diabetes (type 2) but it is strange that type 1 diabetes (insulin dependent) is also increasing and type 1 diabetes is not associated with obesity.

Diabetes, whether it is type 1 or type 2 , is a disease of insulin deficiency. Insulin is secreted by the $B$ cells situated in the islets of Langerhans in the pancreas. In type 1 diabetes the deficiency is so great that insulin is the only treatment that will stop the death of the patient. The $B$ cells have completely or almost completely failed. In type 2 diabetes, to begin with there is excess insulin but there is resistance to the actions of insulin, hence the high blood sugars. In fact, hyperinsulinaemia is found before the blood sugar rises into the diabetes range. There is a continuum from normality, going through the phase of pre-diabetes, to diabetes. This is the result of blood sugar being used to define diabetes and the definition is an arbitrary one and the committees change the goal posts from time to time. Thus, it is not surprising that the burden of atherosclerosis rises from high normal blood sugars to blood sugars in the diabetes range. Similarly, as serum insulin rises in obesity denoting insulin resistance, cardiovascular risk also increases. Therefore, prediabetes is a good time to start intervention to reduce risk of atherosclerosis. As time goes by the insulin drops and the blood sugars rise further in spite of increasing tablets to stimulate insulin secretion and tablets to improve insulin function. Finally, the type 2 diabetic patient will need insulin. That is if the patient survives long enough.

100 years ago, insulin was discovered [2] and many lives were saved. But then it became apparent that there were complications of diabetes due to high blood sugars. The small arteries were damaged and resulted in blindness, renal failure and neuropathy, leading to ulceration, infection and amputation. The large vessels also were damaged, leading to myocardial infarction, stroke and large vessel peripheral disease, leading to amputation.

Diabetes is very often associated with dyslipidaemia which also influences atherosclerosis development. Shafir and Raz [3] many years ago, showed that abnormality in lipid metabolism preceded hyperglycaemia. It therefore is probable that the start of atherosclerosis is already present when hyperglycaemia emerges. Hypertension is common in diabetic patients, another atherosclerotic risk factor. Finally, obese patients tend to exercise less, and exercise plays a huge role in keeping arteries healthy $[4,5]$.

The only treatment for type 1 diabetes before the discovery of insulin was starvation. What must it have been like to see your child starved to death as even with starvation death was eventually the outcome?

Type 2 diabetes arrives slowly. The phases are first hyperinsulinaemia, then a rise in free fatty acids, followed by a slow rise in blood sugar which frequently is not recognised by the patient and, when diagnosis is made the patient already has complications of the disease. Hence the drive for early diagnosis and treatment, for it is the high blood sugar, high cholesterol and high blood pressure that so damage the arteries. 
Obesity is a driver of diabetes, hypertension and dyslipidaemia. We know this because of the effects of treatments to reduce obesity. Bariatric surgery, now renamed metabolic surgery as it sounds less barbaric, has been devised to reduce weight and is very effective. Many studies have shown that reducing the size of the stomach and bypassing the first part of the duodenum reduces apatite and results in considerable weight loss in the majority of patients [6]. There are side effects and complications, but many obese patients accept the risk as this is the only form of treatment open to them. Now we know many of the mechanisms leading to the weight loss. The stomach and duodenum produce hormones that affect hunger and satiety. For example, ghrelin in the stomach responds to distension by supressing apatite but has other effects as well $[7,8]$. The apatite centre is found in the hypothalamus and it is well known that damage to the hypothalamus may cause severe obesity. In the duodenum, hunger signals include oxyntomodulin, a gut hormone that reduces food intake. There has been modest weight loss in early clinical studies [9]. Trials in combination of oxyntomodulin, GLP-1 and peptide YY have recently also been reported but not impressive as so many hormones are involved in weight maintenance $[10,11]$. It should be remembered that most people keep their early adult weight throughout their lives, thus the regulation of weight is an amazingly intricate system. GLP1 analogies have been developed to stimulate insulin secretion and lower blood sugars in diabetic patients. Semaglutide is perhaps the most potent in weight reduction and can now be given by mouth [12]. GLP-1 has many effects. It slows gastric emptying and therefore increases satiety but it also has central effects on apatite. It is effective in stimulating insulin production in a glucose dependent manner so no risk of hypoglycaemia. The L cells in the distal ilium that produce GLP-1 are stimulated by cholecystokinin from the bile. Leptin is a major player in weight maintenance. Secreted by the adipocyte it has a major effect on apatite. Leptin deficiency, which may be due to genetic abnormality in structure or to receptor dysfunction, is associated with sever hunger and severe obesity. These patients are very rare but can be treated by leptin injections. In most cases of obesity leptin is high not low and the apatite centre is not responsive to leptin injections [13].

The bariatric surgery in most patients results in weight reduction. This often results in reversal of diabetes, reduced cholesterol and reduced blood pressure. An amazing metabolic change. Then came Taylor's group who showed that severe calorie reduction replicated the results of bariatric surgery, reduced liver fat, reduced insulin resistance and reduced fat in the pancreas [14]. The fat in the pancreas is of special interest because fat is associated with increased cholesterol, increased oxidation and free radical production, and damage to the B cell [15]. Weight reduction not only reduces insulin resistance but enhances insulin production. Taylor's $[16,17]$ group went on to show that patients with obesity and diabetes were able to adhere to a low calory diet for a year and in many cases, loose a significant amount of weight, reverse diabetes and reduce hypertension and dyslipidaemia. The new excitement is the finding that a GLP-1 agonist, in this case liraglutide, was successful in maintaining significant weight loss after a short spell of severe calorie restriction [5, 18].

Obesity disturbs lipid homeostasis. Excess calories are converted to fat and stored in the periphery through lipoprotein lipase. But there is a limit, and when that limit has been reached, the triglycerides, the large lipid filled particles which also contain cholesterol, get stuck in the liver and the fatty liver ensues. The fatty liver and the increased fat in the muscle all lead to insulin resistance and drive blood sugars up. The inflammation of the fat in the liver leads to fibrosis and then cirrhosis. The high triglycerides and high number of lipid particles lead to cholesterol deposits in the artery 
and stimulate inflammation, fatty streeks and the atheroma [17]. In diabetes and pre-diabetes the results of blood pressure control are impressive in preventing myocardial events. Statins and fibrates for the treatment of dyslipidaemia are equally impressive and now isopentanoic acid, a vitamin E analogue, also significantly reduced atherosclerotic events [19].

\section{Exercise}

Exercise has many metabolic effects. Exercise improves insulin resistance and lowers blood sugar but also cholesterol and blood pressure, so the treatment of diabetes and obesity is multifactorial. The Steno hospital, some years ago, showed very major benefits of looking after diabetes in a multidisciplinary way, ensuring that blood sugar, blood pressure, cholesterol, attention to exercise and cigarette smoking, made a large difference in outcome in a very short period of time [4].

The value of exercise as part of the strategy to prevent both the metabolic syndrome and diabetes has been well researched. A large population survey in Korea with almost 37,000 people showed that Korean adults who do not engage in exercise had a 1 to 3 times the chance of having Metabolic risks [20]. Resistance exercise, aerobic exercise or both in dieting obese older adults has been examined by Villareal [21]. The physical performance test score increased more in the combination group Body weight reduced in both groups by $9 \%$. Brellenthin et al. [22] showed that resistance exercise was associated with a significant reduced risk of obesity even after considering aerobic exercise. However, both exercises together were associated with least risk of obesity

Interestingly physical activity is different from exercise. Physical activity refers to any bodily movement produced by skeletal muscles that results in expenditure of energy (expressed in kilocalories) and which includes a broad range of occupational, leisure and daily activities. Exercise instead refers to planned or structured physical activity performed for a reason, which can be aerobic exercise, resistance training or combined aerobic and resistance training [23]. In a meta analysis 13 published investigations and 2 review articles Mann et al. [23] came to the conclusion that physical activity and exercise can be utilised to improve cholesterol levels. More intensive exercise is needed to lower LDL cholesterol and triglycerides as compared to raising HDL cholesterol. The dose response relationship between the lipid profile and mode of exercise seems to transcend the mode of exercise. Resistance training may be particularly useful for the elderly [24].

Weight reduction can only be achieved by reduction in calorie intake and/or increase in exercise. Over the years there have been very many diets recommended for diabetes and for weight reduction. Each one has failed, only to be replaced by another. Clinics to help patients to lose weight, using the latest diet have flourished with no suggestion that one diet is any more effective than another. It appears that calorie restriction is necessary! The quality of the calories seems to be immaterial. The ketogenic diet had gained traction but has now gone out of fashion since recent excellent studies have shown it to be no more effective than other forms of calorie restriction. Fitzpatrick et al. [25] showed that at 1-year the ketogenic diet was no better than any other form of calorie restriction. The NLA scientific statement [25] examined current evidence and clinical recommendations on the effects of low and very low carbohydrate (including ketogenic) diets for the management of body weight and other cardiometabolic risk factors. Their conclusion was that there is no demonstrable benefit of these diet as compared to low calorie diets consisting of normal macronutrients in the long term and they draw attention to the dangers of ketogenic diets. 
A systemic review by Chawla s et al. [26] on the effects of low-fat and low-carbohydrate diets on weight loss and lipid levels found that low carbohydrate diets are effective in improving weight loss HDL and Triglyceride profiles but must be balanced with potential consequences of raised LDL and total cholesterol in the long term.

Atherosclerosis is an inflammatory condition. Damage to the artery is driven by the raised blood sugar, blood pressure and raised cholesterol including triglycerides. The mechanism is through an increase in free radicle production. The plaque becomes calcified and there is a lot of recent interest in the part osteopontin might play [27].

Intermittent fasting has also been suggested as an improved way to lose weight with no evidence that this is better than just calorie restriction $[28,29]$. Dietary Supplements have no effect on weight loss but are important in patients who are on long-term weight loss diets and those who have had bariatric surgery. Taylor's group [19] for example, used the counterweight programmed delivery diet which included a multivitamin, multinutrient, complete commercial supplement with no suggestion that these supplements would have any effect on weight loss [16].

Exercise by itself has little impact on weight but with calorie restriction is more effective than calorie restriction alone [30]. Although moderate exercise increases appetite, more intensive exercise has many advantages. For example, the feeling of wellbeing, cardiovascular improvement, improvement in insulin resistance, lowering of cholesterol and increase in HDL cholesterol which are all seen as advantages. Exercise lowers blood pressure and improves blood sugar control. Strenuous exercise for more than 4 hours a day certainly causes weight loss (there are few obese marathon runners) but moderate exercise is not very effective in weight loss as it increases appetite.

\section{The Microbiome}

The microbiome is still being investigated as an important organ in disease. It is not surprising therefore that many attempts have been made to link changes in the microbiome with obesity and diet may change the microbiota and change function [31]. The evidence that the microbiome is the cause of or even a factor in the disturbance of the apatite centre is very weak. In an excellent review Aoun et al. [32] discuss the influence of the gut microbiome on obesity in adults. A good section on probiotics and prebiotics makes it clear that changes found in the microbiota are still associations and little sound evidence exists that changes found are any more than associations. The review ends with the suggestion that further studies are needed to determine the ideal formulation of supplements and to identify the population who might benefit from gut microbiome modulation.

In conclusion, cardiovascular damage in obesity/diabetes has been recognised as being the major reason for early mortality in these patients. The understanding of the mechanisms involved have been clearly defined. Treatment is available to halt progression. Prevention of disease in those who are kept under surveillance makes early detection and early intervention treatment a possibility.

\section{Author Contributions}

Gerald H Tomkin and Daphne Owens contributed equally to the article.

\section{Competing Interests}

The authors have declared that no competing interests exist. 


\section{References}

1. Mokdad AH, Ford ES, Bowman BA, Dietz WH, Vinicor F, Bales VS, et al. Prevalence of obesity, diabetes, and obesity-related health risk factors, 2001. JAMA. 2003; 289: 76-79.

2. Tomkin GH. The centenary celebrations-insulin and diabetes. Int J Diabetes Endocrinol. 2021. Doi: 10.46715/ijde2021.05.1000113.

3. Shafrir E, Raz I. Diabetes: Mellitus or lipidus? Diabetologia. 2003; 46: 433-440.

4. Gæde $P$, Lund-Andersen $\mathrm{H}$, Parving $\mathrm{HH}$, Pedersen O. Effect of a multifactorial intervention on mortality in type 2 diabetes. N Engl J Med. 2008; 358: 580-591.

5. Tomkin GH. Obesity, diabetes and the changing environment. EC Nutrition. 2020: 01-08.

6. Genser $L$, Barrat $C$. Long term outcomes after bariatric and metabolic surgery. Presse Medicale. 2018; 47: 471-479.

7. Schwartz MW, Woods SC, Porte D, Seeley RJ, Baskin DG. Central nervous system control of food intake. Nature. 2000; 404: 661-671.

8. Özcan B, Delhanty PJ, Huisman M, Visser JA, Neggers SJ, van der Lely AJ. Overweight and obesity in type 1 diabetes is not associated with higher ghrelin concentrations. Diabetol Metab Syndr. 2021; 13: 79.

9. Wynne K, Park AJ, Small CJ, Patterson M, Ellis SM, Murphy KG, et al. Subcutaneous oxyntomodulin reduces body weight in overweight and obese subjects: A double-blind, randomized, controlled trial. Diabetes. 2005; 54: 2390-2395.

10. Behary P, Tharakan G, Alexiadou K, Johnson N, Albrechtsen NJ, Kenkre J, et al. Combined GLP1, oxyntomodulin, and peptide $Y Y$ improves body weight and glycemia in obesity and prediabetes/type 2 diabetes: A randomized, single-blinded, placebo-controlled study. Diabetes Care. 2019; 42: 1446-1453.

11. Tack J, Verbeure W, Mori H, Schol J, Van den Houte K, Huang IH, et al. The gastrointestinal tract in hunger and satiety signalling. United European Gastroenterol J. 2021; 9: 727-734.

12. Rizzo M, Nauck MA, Mantzoros CS. Incretin-based therapies in 2021-current status and perspectives for the future. Metab Clin Exp. 2021; 122: 154843.

13. Friedman JM. Leptin and the endocrine control of energy balance. Nat Metab. 2019; 1: 754764.

14. Lean M, Brosnahan N, McLoone P, McCombie L, Higgs AB, Ross $H$, et al. Feasibility and indicative results from a 12-month low-energy liquid diet treatment and maintenance programme for severe obesity. Br J Gen Pract. 2013; 63: e115-e124.

15. Taylor R, Al-Mrabeh A, Zhyzhneuskaya S, Peters C, Barnes AC, Aribisala BS, et al. Remission of human type 2 diabetes requires decrease in liver and pancreas fat content but is dependent upon capacity for $\beta$ cell recovery. Cell Metab. 2018; 28: 547-556.

16. Taylor R, Ramachandran A, Yancy WS, Forouhi NG. Nutritional basis of type 2 diabetes remission. BMJ. 2021; 374: n1449.

17. Lean ME, Leslie WS, Barnes AC, Brosnahan N, Thom G, McCombie L, et al. Primary care-led weight management for remission of type 2 diabetes (DiRECT): An open-label, clusterrandomised trial. Lancet. 2018; 391: 541-551.

18. Lundgren JR, Janus $C$, Jensen SB, Juhl CR, Olsen LM, Christensen RM, et al. Healthy weight loss maintenance with exercise, liraglutide, or both combined. N Engl J Med. 2021; 384: 1719-1730. 
19. Bhatt DL, Steg PG, Miller M, Brinton EA, Jacobson TA, Ketchum SB, et al. Cardiovascular risk reduction with icosapent ethyl for hypertriglyceridemia. N Engl J Med. 2019; 380: 11-22.

20. Cho JH, Ko J, Lim ST. Relationship between metabolic syndrome and moderate-to-vigorous physical activity among adults 18 years old and over. PloS ONE. 2021; 16: e0258097.

21. Villareal DT, Aguirre L, Gurney AB, Waters DL, Sinacore DR, Colombo E, et al. Aerobic or resistance exercise, or both, in dieting obese older adults. N Engl J Med. 2017; 376: 1943-1955.

22. Brellenthin AG, Lee DC, Bennie JA, Sui X, Blair SN. Resistance exercise, alone and in combination with aerobic exercise, and obesity in Dallas, Texas, US: A prospective cohort study. PloS Med. 2021; 18: e1003687.

23. Mann S, Beedie C, Jimenez A. Differential effects of aerobic exercise, resistance training and combined exercise modalities on cholesterol and the lipid profile: Review, synthesis and recommendations. Sports Med. 2014; 44: 211-221.

24. Mcleod JC, Stokes T, Phillips SM. Resistance exercise training as a primary countermeasure to age-related chronic disease. Front Physiol. 2019; 10: 645.

25. Kirkpatrick CF, Bolick JP, Kris-Etherton PM, Sikand G, Aspry KE, Soffer DE, et al. Review of current evidence and clinical recommendations on the effects of low-carbohydrate and very-lowcarbohydrate (including ketogenic) diets for the management of body weight and other cardiometabolic risk factors: A scientific statement from the National Lipid Association Nutrition and Lifestyle Task Force. J Clin Lipidol. 2019; 13: 689-711.

26. Chawla S, Tessarolo Silva F, Amaral Medeiros S, Mekary RA, Radenkovic D. The effect of low-fat and low-carbohydrate diets on weight loss and lipid levels: A systematic review and metaanalysis. Nutrients. 2020; 12: 3774.

27. Yee Lok ZS, Lyle AN. Osteopontin in vascular disease: Friend or foe. Arterioscler Thromb Vasc Biol. 2019; 39: 613-622.

28. de Cabo R, Mattson MP. Effects of intermittent fasting on health, aging, and disease. N Engl J Med. 2019; 381: 2541-2551.

29. Rynders CA, Thomas EA, Zaman A, Pan Z, Catenacci VA, Melanson EL. Effectiveness of intermittent fasting and time-restricted feeding compared to continuous energy restriction for weight loss. Nutrients. 2019; 11: 2442.

30. Orozco LJ, Buchleitner AM, Gimenez-Perez G, i Figuls MR, Richter B, Mauricio D. Exercise or exercise and diet for preventing type 2 diabetes mellitus. Cochrane Database Syst Rev. 2008. Doi: 10.1002/14651858.CD003054.

31. Guirro M, Costa A, Gual-Grau A, Herrero P, Torrell H, Canela N, et al. Effects from diet-induced gut microbiota dysbiosis and obesity can be ameliorated by fecal microbiota transplantation: $A$ multiomics approach. PLoS ONE. 2019; 14: e0218143.

32. Aoun A, Darwish F, Hamod N. The influence of the gut microbiome on obesity in adults and the role of probiotics, prebiotics, and synbiotics for weight loss. Prev Nutr Food Sci. 2020; 25: 113 123. 
OBM Integrative and Complementary Medicine 2021; 6(4), doi:10.21926/obm.icm.2104048

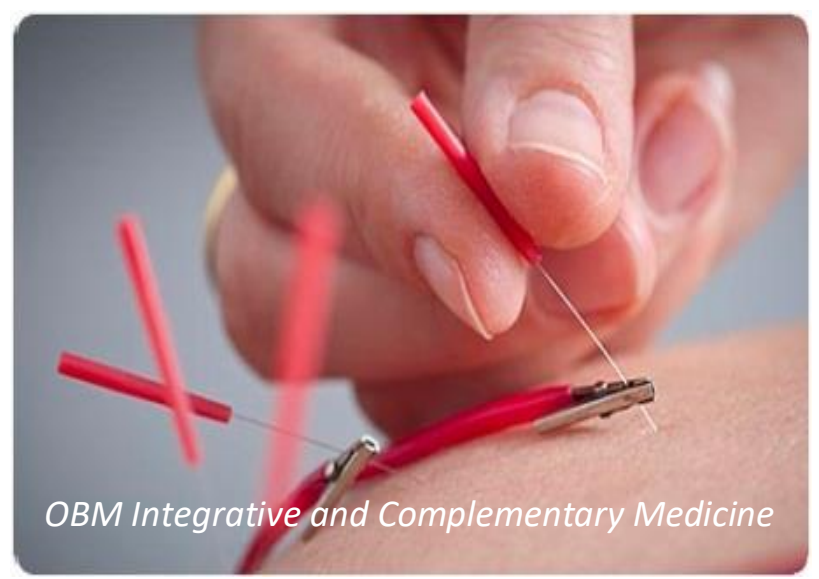

Enjoy $O B M$ Integrative and Complementary Medicine by:

1. Submitting a manuscript

2. Joining in volunteer reviewer bank

3. Joining Editorial Board

4. Guest editing a special issue

For more details, please visit:

http://www.lidsen.com/journals/icm 